\title{
Síndrome de martillo hipotenar: reporte de un caso
}

\author{
Hypothenar hammer syndrome: case report
}

\begin{abstract}
Mariano Revale', Julián Kemps ${ }^{1}$, Sergio Caldiroli', Martín Bodoira' ${ }^{1}$, Antonio Pocoví
\section{RESUMEN}

El síndrome del martillo hipotenar es una entidad poco común caracterizada por la presentación de isquemia digital resultado del trauma repetitivo sobre la eminencia hipotenar de la mano. Ocurre habitualmente en personas que utilizan regularmente sus manos como "martillo" para golpear objetos. Se han descripto diversas estrategias terapéuticas y medidas preventivas para mejorar su evolución a largo plazo. La baja casuística hallada en la literatura está relacionada al subdiagnóstico de esta patología. En esta ocasión se presenta un caso de síndrome de martillo hipotenar recientemente diagnosticado en nuestro medio, y se discute su patogenia, diagnósti-

ABSTRACT

The hypothenar hammer syndrome is an entity characterized by the presentation of digital ischemia resulting from repetitive trauma on the hypothenar eminence of the hand. It usually occurs in people who regularly use their hands as a "hammer" to push, grind or twist objects. Various therapeutics strategies and preventive methods have been described to improve their long-term evolution. The low casuistry found in the literature is related to the subdiagnosis of this pathology. In this report we present a case of hypothenar hammer syndrome recently diagnosed in our environment, and discuss its pathogenesis, diagnosis and treatment.
\end{abstract} co y tratamiento.

Palabras claves: sindrome martillo hipotenar; isquemia digital; traumatismo hipotenar repetitivo; oclusión de arteria cubital; fenómeno de Raynaud.
Key words: hypothenar hammer syndrome; digital ischemia; repetitive hypothenar trauma; ulnar artery occlusion; Raynaud's phenomenon.

Revista Argentina de Cardioangiología Intervencionista 2018;9(2):103-105. Doi: 10.30567/RACI/201802/0103-0105

\section{INTRODUCCIÓN}

Paciente de 27 años, sexo masculino, de oficio carpintero, sin historia de enfermedades relevantes previas, consulta a la guardia por dolor, alteraciones sensitivas y cambios de coloración de su mano derecha durante los últimos 5 días. Relata un episodio compatible con fenómeno de Raynaud en dicha extremidad el mes previo.

Al examen físico se presenta con cianosis, lesiones eritematosas y retardo del relleno capilar, en especial su 4to dedo, asociado a hipoestesia y paresia moderada del mismo (Figuras 1 y 2). Como hallazgo particular presentaba una masa pulsátil en la región hipotenar palmar. Por tal motivo se decidió realizar una angiografía selectiva que evidenció un aneurisma de la arteria cubital, con arcos palmares incompletos, y evidencia de marcado hipoflujo en los ramos digitales palmares comunes y propios predominantemente en los dedos 3ro, 4 to y 5 to. (Figura 3).

Luego de una discusión multidisciplinaria, se decidió adoptar conducta quirúrgica lográndose la completa escisión del aneurisma y anastomosis término-terminal arterial, la cual fue efectuada sin complicaciones (Figura 4).

El paciente evolucionó con recuperación total de la funcionalidad y sensibilidad de su mano derecha y fue dado de alta a las 48 horas luego del procedimiento.

\section{DISCUSIÓN}

El sindrome de martillo hipotenar (SMH) es una patología causada por la lesión vascular traumática de la arteria cubital (ulnar) asociada al uso reiterado de la región hipotenar

1. Sanatorio de la Trinidad Mitre, CABA

Sanatorio San Lucas, Provincia de Buenos Aires

$\triangle$ Correspondencia: Dr. Mariano Andrés Revale. Av. Entre Ríos $6407 m o$ "28", C1080ABN CABA, Rep. Argentina.marianorevale@gmail.com

Los autores no declaran conflictos de intereses

Recibido: 29/04/2018 |Aceptado: 11/05/2018 palmar para impactar sobre un objeto. Esta entidad, inicialmente descripta por Guttani y Von Rosen, fue estudiada décadas más tarde por Conn, quien observó que el mecanismo fisiopatológico subyacente en todos los casos presentados era el traumatismo de la arteria cubital, otorgándole así su denominación.

La arteria cubital es más susceptible a lesiones dado que transcurre superficialmente sobre el ligamento transverso del carpo y los músculos de la region hipotenar, entre los huesos ganchoso y pisiforme.

Anatómicamente, la arteria radial aporta la irrigación del arco palmar profundo, y la arteria cubital la del arco palmar superficial. En la mayoría de los pacientes existe suficiente circulación colateral entre dichas arterias pero en algunos casos los arcos palmares pueden ser "incompletos”. El SMH en estos últimos suele presentarse clí-

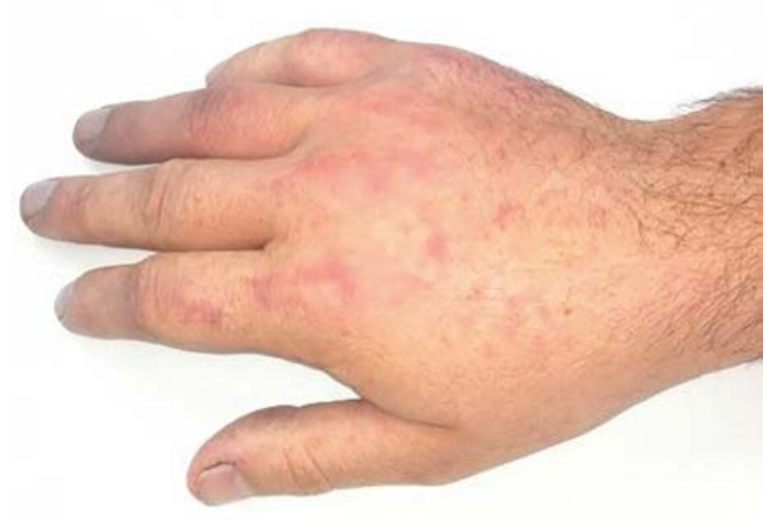

Figura 1. Foto del dorso de la mano comprometida clínicamente. Se evidencian signos de isquemia distal de los dedos. 


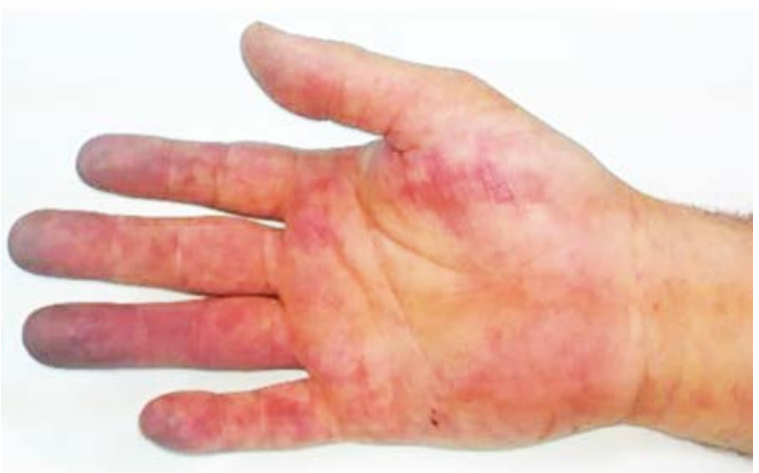

Figura 2. Foto de la palma de la mano comprometida clínicamente. Se evidencian signos de isquemia distal de los dedos.

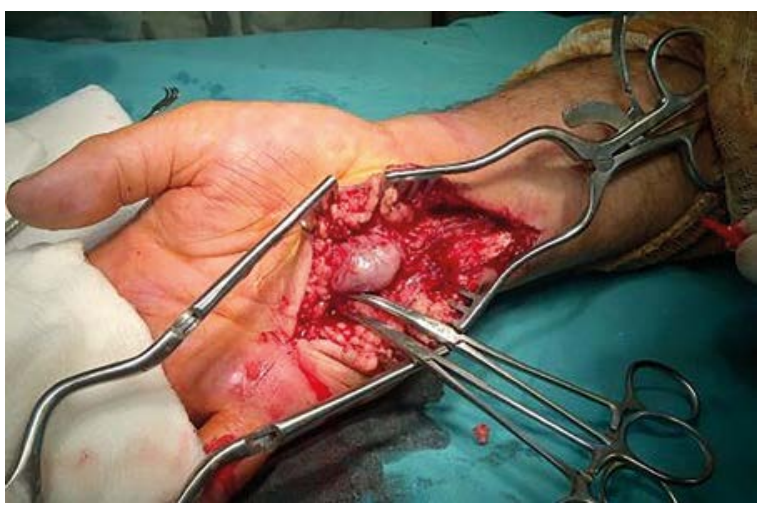

Figura 4. Foto intraoperatoria donde se observa el aneurisma de la arteria cubital previo a su resección.

nicamente de manera más severa requiriendo frecuentemente resolución quirúrgica.

Habitualmente, la duración de ese estímulo traumático sobre la arteria cubital, que se ve asociado a ciertos oficios de riesgo (tales como carpinteros, mecánicos, marroquineros, mineros, maquinistas, obreros) y a ciertos deportes (pelota vasca, tenis, baseball, mountain bike), está directamente relacionada con la aparición de esta patología ${ }^{1}$.

\section{DIAGNÓSTICO}

Habitualmente afecta a hombres de mediana edad (media de 40 años), tabaquistas, que se presentan con frialdad de los dedos de la mano dominante (38\%), intolerancia al frío (72\%), disestesias dolorosas (26\%), entumecimiento (28\%), dolor (22\%), úlceras (15\%) y masa pulsátil (4\%).

El test de Allen es una prueba útil, al igual que el eco-Doppler color, para evaluar la competencia del arco palmar. Sin embargo, el diagnóstico definitivo (gold-standard) es establecido por angiografía arterial selectiva. Las imágenes patognomónicas de esta patología son conocidas como "arteria en forma de sacacorchos”, causadas por la importante tortuosidad de la arteria cubital en esta región, asociadas a su vez, aunque menos frecuentemente, a la visualización de aneurismas, oclusión de la arteria cubital en la región del

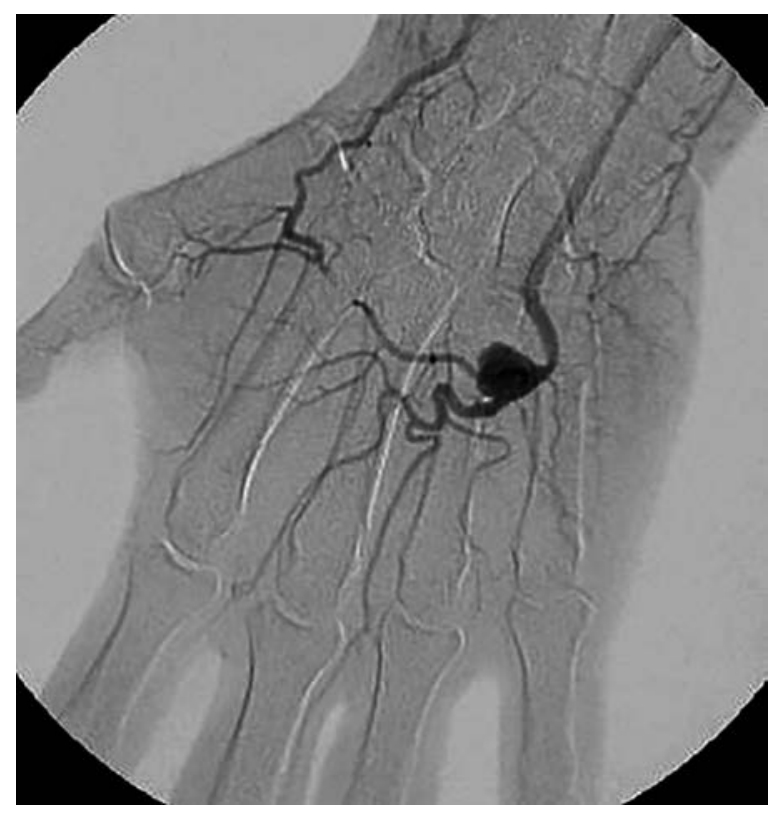

Figura 3. Angiografía de la mano comprometida clínicamente. Se observa una dilatación aneurismática en la arteria cubital, con arco palmar incompleto, marcado hipoflujo de los ramos digitales palmares comunes y propios, predominantemente del $3 e r, 4$ to y 5 to dedo.

hueso ganchoso, y oclusión de arterias digitales subsidiarias de la arteria principal comprometida ${ }^{2}$.

Los diagnósticos diferenciales suelen ser patologías que cursan con signos de isquemia de una extremidad superior incluyendo la enfermedad de Raynaud, vasculitis, embolia arterial de origen cardíaco, tromboangeítis obliterante, aterosclerosis con trombosis secundaria y síndrome del opérculo torácico.

\section{FISIOPATOLOGÍA.}

El mecanismo fisiopatológico de esta entidad todavía está en discusión dada la baja prevalencia de la misma, pero se cree que el traumatismo repetitivo del arco palmar superficial de la arteria cubital podría generar daños en su capa íntima desencadenando mediadores que estimulan el vasoespasmo e induciendo a la activación y la agregación plaquetaria y la formación de trombos. Si el daño se extiende hasta la capa media podría predisponer al debilitamiento de la pared, fibrosis y remodelamiento y el consiguiente desarrollo de aneurismas localizados que actúan como potencial fuente embolígena ${ }^{3,4}$.

Por otro lado, los estudios histopatológicos muestran patrones de displasia fibromuscular en todos los casos evaluados. Este dato, sumado a que el $98 \%$ de los pacientes presentan lesiones angiográficas similares (displasia fibromuscular) en ambas manos a pesar de no ser sintomáticas, hace suponer la presencia de un componente congénito predisponente ${ }^{5}$.

\section{TRATAMIENTO}

La estrategia terapéutica es controversial debido de la escasa casuística.

El tratamiento conservador incluye la cesación tabáquica, la prohibición de continuar con actividades que predisponen a nuevos traumas sobre la región (considerar el cambio de oficio), el uso de guantes protectores, evitar el frío extremo, la administración de bloqueantes cálcicos (nifedipi- 
na, diltiazem) como prevención del espasmo arterial, el tratamiento con antiagregantes o anticoagulantes, cuidados locales en caso de necrosis, y pentoxifilina como vasodilatador y agente reoactivo ${ }^{6}$.

La alternativa quirúrgica puede ser necesaria para el tratamiento de isquemia severa o refractaria, y en contexto de aneurismas arteriales.

Incluye desde la ligadura de la arteria (asumiendo un arco palmar intacto y en casos donde la reconstrucción es técnicamente imposible de realizar), la resección del segmento arterial trombosado o del aneurisma, con anastomosis término-terminal (cuando la resección es menor de $2 \mathrm{~cm}$ de longitud y los trayectos más distales del arco palmar superficial se encuentranen buen estado), hasta la resección y reconstrucción vascular con vena o prótesis en caso de ser necesario ${ }^{7}$.

Generalmente el SMH es diagnosticado demasiado tarde para que la recanalización sea viable, aunque la resección seguida de la interposición de un bypass venoso con la restitución funcional ha sido reportada en varias oportunidades, con un $84 \%$ de permeabilidad de los mismos a 2 años de seguimiento. La oclusión de los puentes ha ocurrido principalmente en pacientes que continuaron con el hábito tabáquico ${ }^{8}$.

La amputación, simpatectomía cervical, el bloqueo del ganglio estrellado y la trombolisis han sido también otros mé- todos alternativos sugeridos, aunque la evidencia al respecto es algo escasa ${ }^{9}$.

Los resultados en el seguimiento de los pacientes con resolución quirúrgica son disímiles, evidenciándose mejoría clínica solo en un $50 \%$ de los casos, sin encontrarse diferencias sustanciales en cuanto a las técnicas empleadas para su revascularización, probablemente debido a que los factores más importante en la evolución a largo plazo sean las medidas higiénico dietéticas y los cuidados preventivos.

\section{CONCLUSIÓN}

El SMH no es una patología tan infrecuente como se cree. Es una causa curable y prevenible de isquemia digital de miembro superior con la que los médicos de atención primaria debieran familiarizarse dado que estudios en poblaciones de riesgo han detectado un alto porcentaje de individuos con evidencia clínica de oclusiones arteriales, con una tasa extremadamente baja de consulta debido a que los síntomas muchas veces no adquieren la suficiente severidad para que interfieran con su actividad laboral diaria.

El SMH es una entidad subdiagnosticada porque su presentación más frecuente es asintomática. Sin embargo, existen casos, como el presentado en este artículo, que requieren resoluciones más invasivas dada la gravedad de su presentación clínica. ${ }^{10}$

\section{BIBLIOGRAFÍA}

1. Little JM, Ferguson DA. The incidence of the hypothenar hammer syndrome. Arch Surg 1972;105:684-5.

2. Ablett CT, Hackett LA. Hypothenar hammer syndrome: case reports and brief review. Clin Med Res 2008;6:3-8.

3. Wernick R, Smith DL. Bilateral hypothenarhammer syndrome: an unusual and preventable cause of digital ischemia. Am J Emerg Med $1989 \mathrm{Ma-}$ y; $7(3): 302 e 306$.

4. Zimmerman NB. Occlusivevascular disorders of the upperextremity. Hand Clin 1993 Feb;9(1):139e150.

5. Perris B, Taylor L, et al. Hypothenar hammer syndrome: Proposed Etiology. J VascSurg2000;31:104-13.

6. Wieczorek I, Farber A, Alexander K. Hypothenar hammer syndrome successfully managed with intravenous prosta- glandin E1 and hepa-

rin with correction of thrombogenic risk profile. A case report. Angiology 1996;47:1111-6.

7. Vayssairat M, Debure C, Cormier JM, Bruneval P, Laurian C, Juillet Y. Hypothenar hammer syndrome: seventeen cases with long-term follow-up. $J$ Vasc Surg 1987;5:838-43.

8. Jagenburg A, Goyen M, Hirschelmann R, Carstens IMM, Kroger K. Hypothenarhammer syndrome: causes, sequelae and diagnostic aspects. Fortschr Geb Rontgenstr Bildgeb 2000;172:295-300.

9. Foster DR, Cameron DC. Hypothenar hammer syndrome. $\mathrm{Br} / \mathrm{Ra}$ diol1981;54:995-996.

10. Abudakka M, Pillai A, Al-Khaffaf H. Hypothenar Hammer Syndrome: rare or underdiagnosed? Eur J Vasc Endovasc Surg 2006;32:257-260. 